\title{
Optimization on the Precision of the MEMS-Redundant IMU Based on Adhesive Joint Assembly
}

\author{
Wanliang Zhao, ${ }^{1,2}$ Xiangyu Sun, ${ }^{1,2}$ Yijie Rong, ${ }^{1,2}$ Jie Duan, ${ }^{1,2}$ Jiawei Chen, ${ }^{1,2}$ Lijun Song $\left(\mathbb{D},{ }^{3}\right.$ \\ and Qinyi Pan ${ }^{1,2}$ \\ ${ }^{1}$ Shanghai Aerospace Control Technology Institute, Shanghai 201100, China \\ ${ }^{2}$ Shanghai Engineering Research Center of Inertia, Shanghai 201100, China \\ ${ }^{3}$ School of Information and Control Engineering, Xi'an University of Architecture and Technology, Xi'an 710055, China
}

Correspondence should be addressed to Lijun Song; songlijun9071@sina.com

Received 28 August 2020; Revised 13 September 2020; Accepted 20 September 2020; Published 9 October 2020

Academic Editor: Tao Zhang

Copyright (c) 2020 Wanliang Zhao et al. This is an open access article distributed under the Creative Commons Attribution License, which permits unrestricted use, distribution, and reproduction in any medium, provided the original work is properly cited.

\begin{abstract}
In order to improve the precision of the spaceborne Inertial Measurement Unit (IMU), this paper proposes an adhesive joint assembly of the MEMS-redundant IMU. That is the application of special redundant installation of multiple MEMS gyroscopes in the IMU, which can improve the reliability of the MEMS-redundant IMU on the basis of reducing the weight of IMU. However, with the change of working environment, the traditional mechanical assembly of MEMS-redundant IMU will produce the large packaging stress and cause the deformation of MEMS gyroscope. This change will lead to changes in installation errors, scale factor errors, and bias errors of the MEMS gyroscope, resulting in a significant reduction in measurement precision of the MEMSredundant IMU. Therefore, this paper selects the adhesive material that matches the thermal physical parameters of the material with the circuit board by analyzing the requirements of MEMS gyroscope on working environment at first. Then, by optimizing the bonding process, the installation error of each axis of MEMS-redundant IMU under different temperatures is better than the traditional mechanical connection mode. The experiment results of thermal vacuum show that the new assembly method can reduce the influence of temperature on the bias. Compared with the traditional method, the new assembly which is based on adhesive joint assembly can improve the measurement precision of MEMS-redundant IMU by an order of magnitude.
\end{abstract}

\section{Introduction}

In modern applications, with the growing demand of nanosatellites, the inertial navigation systems are developing towards the direction of low cost, miniaturization, and low power consumption [1,2]. Micro electro mechanical system (MEMS) gyroscope is made of silicon semiconductor materials by advanced micro/nano technology and ASIC manufacturing technology. It has been widely used with advantages of small size, low cost, and low power, etc. [3, 4].

As the core measurement component of microinertial navigation systems, gyroscopes and accelerometers of the IMU are susceptible to factors such as component aging, improper operation, and interference of harsh working conditions. As a result, the measurement precision of gyroscope and accelerometer declines or even fails to work normally $[5,6]$.

Redundancy technology is an effective method to improve the reliability of system, and widely used in aviation and aerospace fields $[7,8]$. The reliability of strap-down inertial navigation system can be improved without improving the performance of a single inertial device. Redundancy technology is divided into two levels, including the system redundancy technology and device redundancy technology. Compared with the system redundancy technology, the device redundancy technology constitutes a MEMS-redundant IMU by increasing the number of inertial devices, which has more advantages in reducing system volume and engineering costs $[9,10]$. 
In MEMS-redundant IMU, the ubiquitous installation errors, scale factor errors, bias, and noises of MEMS gyroscope have a certain impact on the precision of gyroscope output and then affect the navigation precision [11]. The error processing technology of MEMS-redundant IMU is developed to improve the measurement and navigation precision of system $[12,13]$.

To reduce the error of measurement and improve the precision of inertial navigation system, the traditional method estimates and compensates the alignment error, scale factor error, and bias of gyroscope in MEMS-redundant IMU by calibration compensation technique [14]. The calibration compensation technology uses turntable or other high-precision auxiliary devices to provide reference value and processes the error of MEMS redundant IMU by mathematical modeling method, which is suitable for deterministic error compensation $[15,16]$. However, in the actual working environment, MEMS gyroscope is easily disturbed by temperature, vibration, and other operating conditions. The resulting uncertain variations of installation error, scale factor error, and bias greatly affect the output precision of MEMS gyroscope, so that the navigation accuracy is reduced.

The precision of MEMS-redundant IMU cannot be assured only by calibration compensation scheme, and the assembly technique of MEMS redundant IMU should be designed to reduce the effect of working environment on the error of MEMS gyroscope $[17,18]$. The MEMS gyroscope is fixed on the sensitive axis by specific packaging technologies to decline the change of IMU error. Therefore, the error of system measurement is minimized to ensure the high navigation precision. Traditional assembly technology of mechanical assembly is mainly used screw, and this way requires circuit board to leave enough space for threaded connection, enlarging the volume of structure. Furthermore, when the temperature changed in the working environment of MEMS redundant IMU, MEMS gyroscope influenced by processing manufacturing and environmental conditions will produce deformation. So, it changes the error of installation and bias and reduces the precision of MEMS redundant IMU $[19,20]$.

In order to decline the influence of working environment on gyroscope and improve the precision of MEMS redundant IMU, an optimization method on the precision of MEMSredundant IMU based on adhesive joint assembly is proposed in this paper. This method abandons the traditional way of mechanically installing gyroscopes and circuit boards.

\section{The Error of MEMS Redundant IMU}

2.1. The Measurement Model of MEMS Redundant IMU. As shown in Figure 1, $o x_{b}, o y_{b}$, and $o z_{b}$ are three axes of the carrier coordinate system. The gyroscope $i$ is installed obliquely in the redundant system. The angle between the measurement axis and the $o z_{b}$ axis is $\alpha_{i}$, and the angle between the projection of gyroscope $i$ on the $x_{b} o y_{b}$ plane and the direction of $o x_{b}$ axis is $\beta_{i}$.

In the redundant system, the output of gyroscope $i$ is

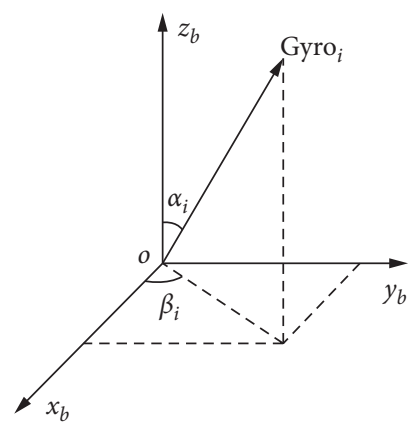

FIGURe 1: Projection of the gyroscope in MEMS-redundant IMU system on the carrier coordinate system.

$$
\begin{aligned}
\omega_{i} & =h_{1}^{i}\left(\alpha_{i}, \beta_{i}\right) \omega_{x}+h_{2}^{i}\left(\alpha_{i}, \beta_{i}\right) \omega_{y}+h_{3}^{i}\left(\alpha_{i}, \beta_{i}\right) \omega_{z} \\
& =\sin \alpha_{i} \cos \beta_{i} \omega_{x}+\sin \alpha_{i} \sin \beta_{i} \omega_{y}+\cos \alpha_{i} \omega_{z}
\end{aligned}
$$

where $\omega_{x}, \omega_{y}, \omega_{z}$ are the angular rates of gyroscope $i$ along the axes of $o x_{b}, o y_{b}$, and $o z_{b} ; h_{1}^{i}, h_{2}^{i}$, and $h_{3}^{i}$ are the projections of gyroscope $i$ in three axes of the body frame, respectively, which are related to the included angles $\alpha_{i}$ and $\beta_{i}$.

Thus, the output of $n$ gyroscopes in the redundant system can be expressed as

$$
\begin{aligned}
Z & =\left[\begin{array}{c}
\omega_{1} \\
\omega_{2} \\
\vdots \\
\omega_{n}
\end{array}\right]=\left[\begin{array}{ccc}
h_{1}^{1} & h_{2}^{1} & h_{3}^{1} \\
h_{1}^{2} & h_{2}^{2} & h_{3}^{2} \\
\vdots & \vdots & \vdots \\
h_{1}^{n} & h_{2}^{n} & h_{3}^{n}
\end{array}\right] \cdot\left[\begin{array}{c}
\omega_{x} \\
\omega_{y} \\
\omega_{z}
\end{array}\right]+\left[\begin{array}{c}
\varepsilon_{1} \\
\varepsilon_{2} \\
\vdots \\
\varepsilon_{n}
\end{array}\right] \\
& =\left[\begin{array}{ccc}
\sin \alpha_{1} \cos \beta_{1} & \sin \alpha_{1} \sin \beta_{1} & \cos \alpha_{1} \\
\sin \alpha_{2} \cos \beta_{2} & \sin \alpha_{2} \sin \beta_{2} & \cos \alpha_{2} \\
\vdots & \vdots & \vdots \\
\sin \alpha_{n} \cos \beta_{n} & \sin \alpha_{n} \sin \beta_{n} & \cos \alpha_{n}
\end{array}\right] \cdot\left[\begin{array}{c}
\omega_{x} \\
\omega_{y} \\
\omega_{z}
\end{array}\right]+\left[\begin{array}{c}
\varepsilon_{1} \\
\varepsilon_{2} \\
\vdots \\
\varepsilon_{n}
\end{array}\right],
\end{aligned}
$$

where $\mathbf{Z}$ is the measurement matrix of gyroscope output of redundant system, and $\varepsilon_{1}, \varepsilon_{2}, \ldots, \varepsilon_{n}$ are gyroscope measurement noise.

Defined $\mathbf{X}=\left[\begin{array}{lll}\omega_{x} & \omega_{y} & \omega_{z}\end{array}\right]^{\mathrm{T}}, \boldsymbol{\varepsilon}=\left[\begin{array}{llll}\varepsilon_{1} & \varepsilon_{2} & \ldots & \varepsilon_{n}\end{array}\right]^{\mathrm{T}}$, and

$$
\mathbf{H}=\left[\begin{array}{ccc}
h_{1}^{1} & h_{2}^{1} & h_{3}^{1} \\
h_{1}^{2} & h_{2}^{2} & h_{3}^{2} \\
\vdots & \vdots & \vdots \\
h_{1}^{n} & h_{2}^{n} & h_{3}^{n}
\end{array}\right] .
$$

Equation (2) becomes as follows:

$$
\mathbf{Z}=\mathbf{H X}+\boldsymbol{\varepsilon}
$$

where $\mathbf{H} \in R^{n \times 3}$ is the redundant system configuration matrix; $\mathbf{X} \in R^{3 \times 1}$ is the body system triaxial measured values; and $\varepsilon \in R^{n \times 1}$ is the measurement noise matrix of redundant system, which is generally assumed to be Gaussian white noise with zero mean. It is subject to

$$
\begin{aligned}
E(\boldsymbol{\varepsilon}) & =0, \\
E\left(\boldsymbol{\varepsilon} \boldsymbol{\varepsilon}^{\mathrm{T}}\right) & =R=\sigma^{2} \mathbf{I}_{n},
\end{aligned}
$$


where $\mathbf{R}$ is the $(n \times n)$ variance matrix and $\mathbf{I}_{n}$ is the $(n \times n)$ unit matrix.

2.2. The Measurement Error Model of MEMS Redundant IMU. There are several errors such as the error of misalignment, scale factor, and bias of the MEMS-redundant IMU. Thus, the measurement output of the MEMS redundant IMU is considered as follows:

$$
\mathbf{Z}_{\text {actual }}=\left(\mathbf{I}+\mathbf{H}_{s e}\right)\left(\mathbf{H}+\mathbf{H}_{m e}\right) \mathbf{X}+\mathbf{b}+\boldsymbol{\varepsilon},
$$

where $\mathbf{H}_{s e} \in R^{n \times n}$ is the scale factor error matrix; $\mathbf{H}_{m e} \in R^{m \times n}$ is the misalignment error matrix; $\mathbf{b}$ is the bias vector; and $\boldsymbol{\varepsilon}$ is the noise. The scale factor error matrix can be expressed as

$$
\mathbf{H}_{s e}=\left[\begin{array}{cccc}
k_{1} & 0 & \cdots & 0 \\
0 & k_{2} & \cdots & 0 \\
\cdots & \cdots & \cdots & \cdots \\
0 & 0 & \cdots & k_{n}
\end{array}\right]
$$

And $k_{i}$ is the scale factor error of each gyroscope.

The assemble error matrix is expressed as follows:

$$
\mathbf{H}_{m e}=\left[\begin{array}{ccc}
\sin \left(\alpha_{1}+\Delta \alpha_{1}\right) \cos \left(\beta_{1}+\Delta \beta_{1}\right) & \sin \left(\alpha_{1}+\widetilde{\alpha}_{1}\right) \sin \left(\beta_{1}+\widetilde{\beta}_{1}\right) & \cos \left(\alpha_{1}+\widetilde{\alpha}_{1}\right) \\
\sin \left(\alpha_{2}+\Delta \alpha_{2}\right) \cos \left(\beta_{2}+\Delta \beta_{2}\right) & \sin \left(\alpha_{2}+\widetilde{\alpha}_{2}\right) \sin \left(\beta_{2}+\widetilde{\beta}_{2}\right) & \cos \left(\alpha_{2}+\widetilde{\alpha}_{2}\right) \\
\vdots & \vdots & \vdots \\
\sin \left(\alpha_{n}+\Delta \alpha_{n}\right) \cos \left(\beta_{n}+\Delta \beta_{n}\right) & \sin \left(\alpha_{n}+\widetilde{\alpha}_{n}\right) \sin \left(\beta_{n}+\widetilde{\beta}_{n}\right) & \cos \left(\alpha_{n}+\widetilde{\alpha}_{n}\right)
\end{array}\right]-\mathbf{H}
$$

where $\Delta \alpha$ and $\Delta \beta$ are the misalignment angles of each gyro.

The bias vector becomes

$$
\mathbf{b}=\left[\begin{array}{llll}
b_{1} & b_{2} & \ldots & b_{n}
\end{array}\right]^{\mathrm{T}} .
$$

In the actual working environment, the error of gyroscope changes because the gyroscope is susceptible to temperature and other factors. The scale factor error matrix, assemble error matrix and bias matrix can be written as

\subsection{The Analysis of the Error of MEMS Redundant IMU.}

$$
\begin{aligned}
& \mathbf{H}_{s e}=\overline{\mathbf{H}}_{s e}+\delta \mathbf{H}_{s e}=\left[\begin{array}{cccc}
k_{1}+\delta k_{1} & 0 & \ldots & 0 \\
0 & k_{2}+\delta k_{2} & \ldots & 0 \\
\ldots & \ldots & \ldots & \ldots \\
0 & 0 & \ldots & k_{n}+\delta k_{n}
\end{array}\right] \\
& \mathbf{H}_{m e}=\overline{\mathbf{H}}_{m e}+\delta \mathbf{H}_{m e} \\
& =\left[\begin{array}{ccc}
\sin \left(\alpha_{1}+\widetilde{\alpha}_{1}+\delta \widetilde{\alpha}_{1}\right) \cos \left(\beta_{1}+\widetilde{\beta}_{1}+\delta \widetilde{\beta}_{1}\right) & \sin \left(\alpha_{1}+\widetilde{\alpha}_{1}+\delta \widetilde{\alpha}_{1}\right) \sin \left(\beta_{1}+\widetilde{\beta}_{1}+\delta \widetilde{\beta}_{1}\right) & \cos \left(\alpha_{1}+\widetilde{\alpha}_{1}+\delta \widetilde{\alpha}_{1}\right) \\
\sin \left(\alpha_{2}+\widetilde{\alpha}_{2}+\delta \widetilde{\alpha}_{2}\right) \cos \left(\beta_{2}+\widetilde{\beta}_{2}+\delta \widetilde{\beta}_{2}\right) & \sin \left(\alpha_{2}+\widetilde{\alpha}_{2}+\delta \widetilde{\alpha}_{2}\right) \sin \left(\beta_{2}+\widetilde{\beta}_{2}+\delta \widetilde{\beta}_{2}\right) & \cos \left(\alpha_{2}+\widetilde{\alpha}_{2}+\delta \widetilde{\alpha}_{2}\right) \\
\vdots & \vdots & \vdots \\
\sin \left(\alpha_{n}+\widetilde{\alpha}_{n}+\delta \widetilde{\alpha}_{n}\right) \cos \left(\beta_{n}+\widetilde{\beta}_{n}+\delta \widetilde{\beta}_{n}\right) & \sin \left(\alpha_{n}+\widetilde{\alpha}_{n}+\delta \widetilde{\alpha}_{n}\right) \sin \left(\beta_{n}+\widetilde{\beta}_{n}+\delta \widetilde{\beta}_{n}\right) & \cos \left(\alpha_{n}+\widetilde{\alpha}_{n}+\delta \widetilde{\alpha}_{n}\right)
\end{array}\right]-\mathbf{H}, \\
& \mathbf{b}=\overline{\mathbf{b}}+\delta \mathbf{b}=\mathbf{b}=\left[\begin{array}{lllll}
b_{1}+\delta b_{1} & b_{2}+\delta b_{2} & \ldots & b_{n}+\delta b_{n}
\end{array}\right]^{\mathrm{T}},
\end{aligned}
$$

where $k$ and $b$ are the scale factor and bias of each gyroscope; $\widetilde{\alpha}$ and $\widetilde{\beta}$ are the misalignment angle of each gyroscope; $\delta k$ and $\delta b$ are the error of scale factor and bias of each gyroscope; and $\delta \widetilde{\alpha}$ and $\delta \widetilde{\beta}$ are the error of misalignment angle of each gyroscope.

In this case, the measurement output of redundant IMU can be calculated by 


$$
\begin{aligned}
& \mathbf{Z}=\left(\mathbf{I}+\overline{\mathbf{H}}_{s e}+\delta \mathbf{H}_{s e}\right)\left(\mathbf{H}+\overline{\mathbf{H}}_{m e}+\delta \mathbf{H}_{m e}\right) \mathbf{X}+\overline{\mathbf{b}}+\delta \mathbf{b}+\boldsymbol{\varepsilon} \\
& \Delta \mathbf{Z}=\mathbf{Z}-\mathbf{Z}_{\text {true }} \\
& =\left(\mathbf{I}+\overline{\mathbf{H}}_{s e}+\delta \mathbf{H}_{s e}\right)\left(\mathbf{H}+\overline{\mathbf{H}}_{m e}+\delta \mathbf{H}_{m e}\right) \mathbf{X}+\overline{\mathbf{b}}+\delta \mathbf{b}+\boldsymbol{\varepsilon}-(\mathbf{H X}+\boldsymbol{\varepsilon}) \\
& =\left[\overline{\mathbf{H}}_{m e}+\delta \mathbf{H}_{m e}+\left(\overline{\mathbf{H}}_{s e}+\delta \mathbf{H}_{s e}\right)\left(\mathbf{H}+\overline{\mathbf{H}}_{m e}+\delta \mathbf{H}_{m e}\right)\right] \mathbf{X}+\overline{\mathbf{b}}+\delta \mathbf{b}+\boldsymbol{\varepsilon} \\
& =\left(\begin{array}{ccc}
\sin \left(\alpha_{1}+\widetilde{\alpha}_{1}+\delta \widetilde{\alpha}_{1}\right) \cos \left(\beta_{1}+\widetilde{\beta}_{1}+\delta \widetilde{\beta}_{1}\right) & \sin \left(\alpha_{1}+\widetilde{\alpha}_{1}+\delta \widetilde{\alpha}_{1}\right) \sin \left(\beta_{1}+\widetilde{\beta}_{1}+\delta \widetilde{\beta}_{1}\right) & \cos \left(\alpha_{1}+\widetilde{\alpha}_{1}+\delta \widetilde{\alpha}_{1}\right) \\
\sin \left(\alpha_{2}+\widetilde{\alpha}_{2}+\delta \widetilde{\alpha}_{2}\right) \cos \left(\beta_{2}+\widetilde{\beta}_{2}+\delta \widetilde{\beta}_{2}\right) & \sin \left(\alpha_{2}+\widetilde{\alpha}_{2}+\delta \widetilde{\alpha}_{2}\right) \sin \left(\beta_{2}+\widetilde{\beta}_{2}+\delta \widetilde{\beta}_{2}\right) & \cos \left(\alpha_{2}+\widetilde{\alpha}_{2}+\delta \widetilde{\alpha}_{2}\right) \\
\vdots & \vdots & \vdots \\
\sin \left(\alpha_{n}+\widetilde{\alpha}_{n}+\delta \widetilde{\alpha}_{n}\right) \cos \left(\beta_{n}+\widetilde{\beta}_{n}+\delta \widetilde{\beta}_{n}\right) & \sin \left(\alpha_{n}+\widetilde{\alpha}_{n}+\delta \widetilde{\alpha}_{n}\right) \sin \left(\beta_{n}+\widetilde{\beta}_{n}+\delta \widetilde{\beta}_{n}\right) & \cos \left(\alpha_{n}+\widetilde{\alpha}_{n}+\delta \widetilde{\alpha}_{n}\right)
\end{array}\right] X \\
& +\left(\left[\begin{array}{cccc}
k_{1}+\delta k_{1} & 0 & \ldots & 0 \\
0 & k_{2}+\delta k_{2} & \ldots & 0 \\
\ldots & \ldots & \ldots & \ldots \\
0 & 0 & \ldots & k_{n}+\delta k_{n}
\end{array}\right]\right. \\
& \left.\cdot\left[\begin{array}{ccc}
\sin \left(\alpha_{1}+\widetilde{\alpha}_{1}+\delta \widetilde{\alpha}_{1}\right) \cos \left(\beta_{1}+\widetilde{\beta}_{1}+\delta \widetilde{\beta}_{1}\right) & \sin \left(\alpha_{1}+\widetilde{\alpha}_{1}+\delta \widetilde{\alpha}_{1}\right) \sin \left(\beta_{1}+\widetilde{\beta}_{1}+\delta \widetilde{\beta}_{1}\right) & \cos \left(\alpha_{1}+\widetilde{\alpha}_{1}+\delta \widetilde{\alpha}_{1}\right) \\
\sin \left(\alpha_{2}+\widetilde{\alpha}_{2}+\delta \widetilde{\alpha}_{2}\right) \cos \left(\beta_{2}+\widetilde{\beta}_{2}+\delta \widetilde{\beta}_{2}\right) & \sin \left(\alpha_{2}+\widetilde{\alpha}_{2}+\delta \widetilde{\alpha}_{2}\right) \sin \left(\beta_{2}+\widetilde{\beta}_{2}+\delta \widetilde{\beta}_{2}\right) & \cos \left(\alpha_{2}+\widetilde{\alpha}_{2}+\delta \widetilde{\alpha}_{2}\right) \\
\vdots & \vdots & \vdots \\
\sin \left(\alpha_{n}+\widetilde{\alpha}_{n}+\delta \widetilde{\alpha}_{n}\right) \cos \left(\beta_{n}+\widetilde{\beta}_{n}+\delta \widetilde{\beta}_{n}\right) & \sin \left(\alpha_{n}+\widetilde{\alpha}_{n}+\delta \widetilde{\alpha}_{n}\right) \sin \left(\beta_{n}+\widetilde{\beta}_{n}+\delta \widetilde{\beta}_{n}\right) & \cos \left(\alpha_{n}+\widetilde{\alpha}_{n}+\delta \widetilde{\alpha}_{n}\right)
\end{array}\right]\right) X \\
& +\left[\begin{array}{llll}
b_{1}+\delta b_{1} & b_{2}+\delta b_{2} & \ldots & b_{n}+\delta b_{n}
\end{array}\right]^{\mathrm{T}}+\varepsilon \text {. }
\end{aligned}
$$

Generally, the gyroscope error can be calibrated by turntable, which has little influence on the output of MEMS-redundant IMU. However, the variation of redundant gyroscope error affected by working environment such as temperature is hard to predict and significantly degrade the MEMS redundant IMU performance and navigation precision.

The simulation is performed to verify the precision of the MEMS redundant IMU affected by the influence of variations of installation error, scale factor error, and bias.

Suppose that the true value of tri-orthogonal axial of the body system is $50^{\circ} / \mathrm{h}$, the noise of each gyroscope is Gaussian white noise, and the standard deviation is $0.1^{\circ} / \mathrm{h}$. The bias of each gyroscope is $0.2^{\circ} / \mathrm{h}$, the misalignment angle is $20^{\prime \prime}$, and the scale factor error is $500 \mathrm{ppm}$. The specific results are as follows.

From Tables 1-3 and Figures 2-4, the error of gyroscope enlarges gradually with the increase of misalignment error, scale-factor error, and bias, and the precision of gyroscope becomes worse. Compared with the descending percentage of gyroscope 1 in Tables 1-3, the change of installation error and scale factor error has relatively minimal impact on the measurement precision among the three errors, and the change of bias has significant influences on the measurement precision. Therefore, the bias which varies with the working environment is the most important factor affecting the measurement precision. By studying the gyroscope adhesive assembly process under thermal vacuum conditions, the change of the gyroscope bias can be reduced, and the measured precision and navigation precision of MEMS redundant IMU can be comprehensively improved.

\section{The Assembly and Manufacturing Process of MEMS Redundant IMU}

The traditional MEMS-IMU assembly structure is illustrated in the Figure 5. The PCB of the MEMS gyroscope assembly is press-mounted on the metal frame by screws, and the upper structure is fixed by the pretightening force between the threads. The pretightening force will be introduced into the MEMS gyroscope because this installation method will lead to irregular warping deformation of PCB board due to the different installation pretightening force of each mounting screw at different temperatures. Even though the constant torque wrench is installed, the warpage and deformation caused by press-mounting is irregular due to the asymmetric arrangement of copper-clad circuit in PCB. When MEMSIMU is subjected to acutely variable temperature, the output of MEMS gyroscope caused by this irregularity is essentially nonlinear, which directly leads to the nonlinear distortion of navigation accuracy. Moreover, after violent vibration, the slight displacement between the PCB and metal frame is irrecoverable, which will cause mismatch between the initial compensation algorithms with the actual position. In order to control the volume and mass of MEMS-IMU, the general screw press-mounting PCB board will be equipped with multiple MEMS gyroscopes, and the error caused by mechanical connection will affect the precision of whole redundancy system.

Aiming at the drawbacks of traditional mechanical assembly, an assembly method of MEMS-redundant IMU based on adhesive joint assembly is proposed in this paper. The schematic diagram of MEMS-redundant IMU assembly structure is as shown in Figure 6. Each MEMS gyroscope is arranged on an isolated PCB board to avoid the error of 
TABLE 1: The precision of gyroscope in different misalignment angle.

\begin{tabular}{lcc}
\hline Misalignment angle (arc-seconds) & Measurement error (deg/hr) & Descending percentage of measurement precision $(\mathrm{deg} / \mathrm{hr})$ \\
\hline 0 & 0.139232 & 0.28 \\
1 & 0.21568 & 0.43 \\
5 & 0.198812 & 0.40 \\
10 & 0.28549 & 0.57 \\
15 & 0.283656 & 0.57 \\
20 & 0.304006 & 0.61 \\
30 & 0.387839 & 0.78 \\
40 & 0.500454 & 1.00 \\
50 & 0.754438 & 1.51 \\
\hline
\end{tabular}

TABle 2: The precision of gyroscope in different scale factor error.

\begin{tabular}{lcc}
\hline Scale factor error $(\mathrm{ppm})$ & Measurement error $(\mathrm{deg} / \mathrm{hr})$ & Descending percentage of measurement precision $(\mathrm{deg} / \mathrm{hr})$ \\
\hline 0 & 0.15338 & 0.31 \\
100 & 0.25276 & 0.51 \\
500 & 0.257578 & 0.52 \\
1000 & 0.1847 & 0.37 \\
2000 & 0.276227 & 0.55 \\
3000 & 0.285564 & 0.57 \\
5000 & 0.346393 & 0.69 \\
10000 & 0.60342 & 1.21 \\
15000 & 0.81882 & 1.64 \\
\hline
\end{tabular}

TABLe 3: The precision of gyroscope in different bias.

\begin{tabular}{lcc}
\hline Bias $(\mathrm{deg} / \mathrm{hr})$ & Measurement error $(\mathrm{deg} / \mathrm{hr})$ & Descending percentage of measurement precision $(\mathrm{deg} / \mathrm{hr})(\%)$ \\
\hline 0 & 0.021749 & 0.04 \\
1 & 1.16887 & 2.34 \\
2 & 2.09351 & 4.19 \\
3 & 3.146141 & 6.29 \\
4 & 4.126136 & 8.25 \\
5 & 4.966945 & 9.93 \\
6 & 5.991852 & 11.98 \\
7 & 6.870689 & 13.74 \\
8 & 7.919157 & 15.84 \\
9 & 8.835051 & 17.67 \\
10 & 9.920551 & 19.84 \\
\hline
\end{tabular}

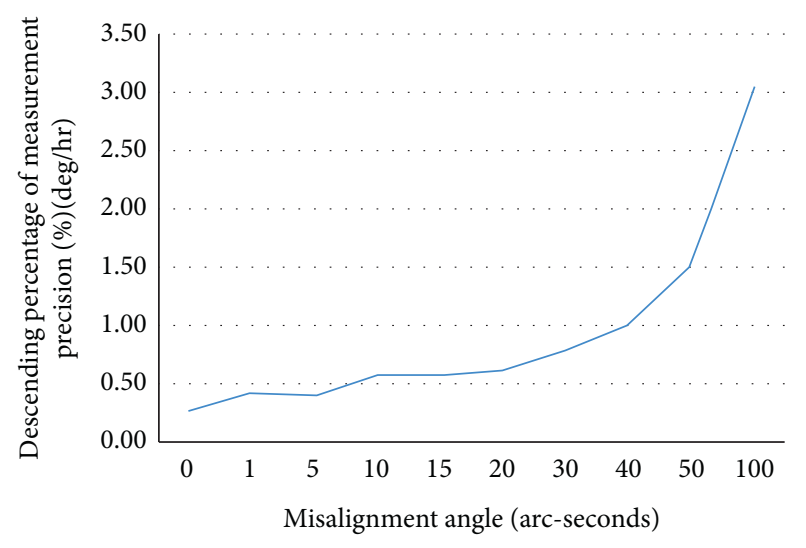

FIgURE 2: The precision of gyroscope in different misalignment angle.

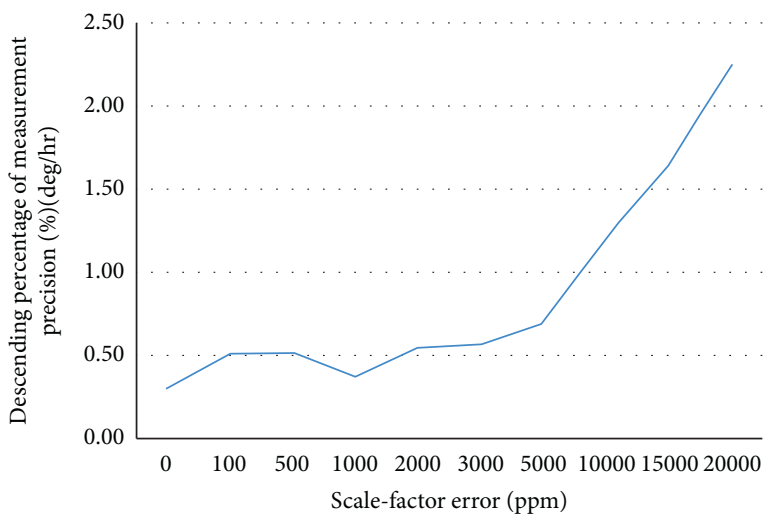

FIGURE 3: The precision of gyroscope in different scale-factor error. 


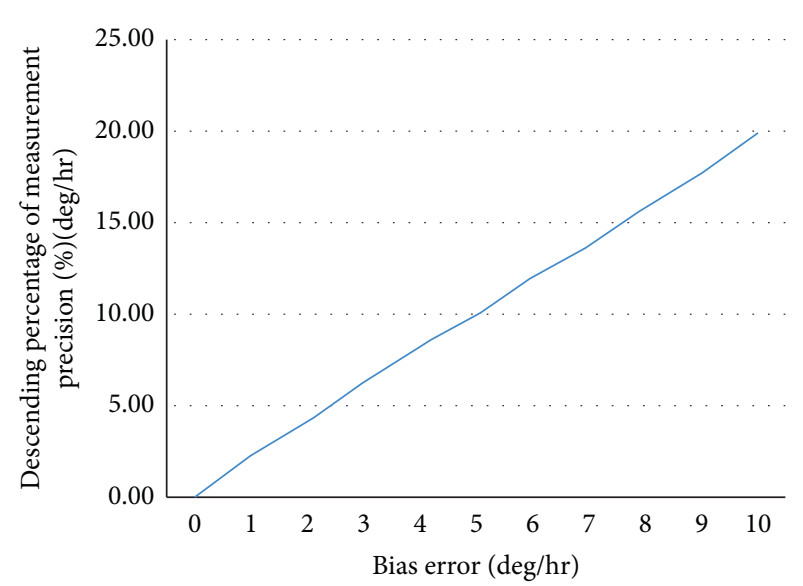

Figure 4: The precision of gyroscope in different bias.

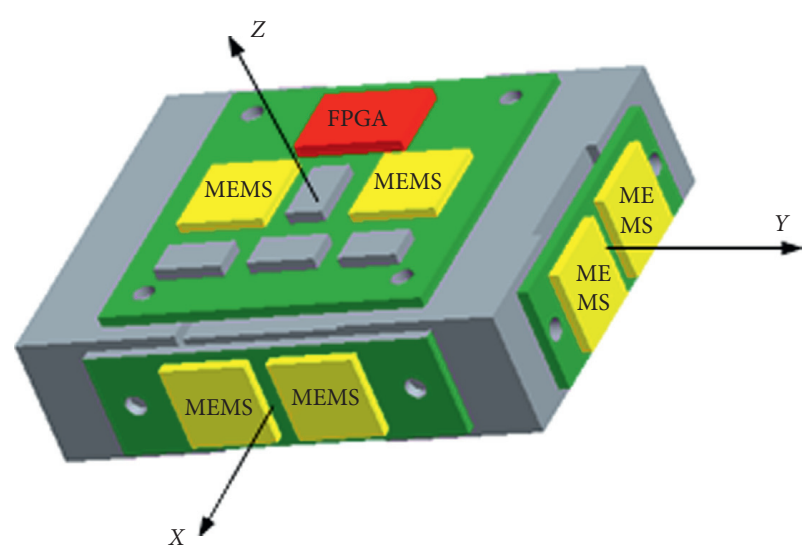

Figure 5: Traditional assembly structure.

cointerference and achieve redundancy. Compared with traditional mechanical assembly method, the overall structure volume is further reduced.

The adhesive joint assembly structure of the single-axis MEMS gyroscope is shown in the Figure 7. The PCB plate is fixed on the metal frame by the adhesive layer. The material of the adhesive layer is a high-temperature curing glue with a thermal expansion coefficient of $\alpha=40 \sim 130 \times 10^{-6} \mathrm{~K}-1$, which perfectly matches the PCB board with thermal expansion coefficient of about $82 \times 10^{-6} \mathrm{~K}-1$. The difference is that in traditional mechanical assembly process, the deformation at the mechanical interface between PCB plate and mounting screw is small with the constraints of the hold-down screw. At the center of PCB equipped with MEMS gyroscope, the warpage deformation is large and enlarged gradually from outside to inside. However, in the adhesive joint assembly process, the stiffness of PCB plate in the warping and deformable area around the MEMS gyroscope is enhanced by controlling the structure of the adhesive layer underneath the PCB. While the peripheral area is designed as the free end, the deformation of PCB plate center is much smaller than that of free end and the deformation expands from inside to outside. It further improves the precision of the MEMS gyroscope under drastically changing temperature.

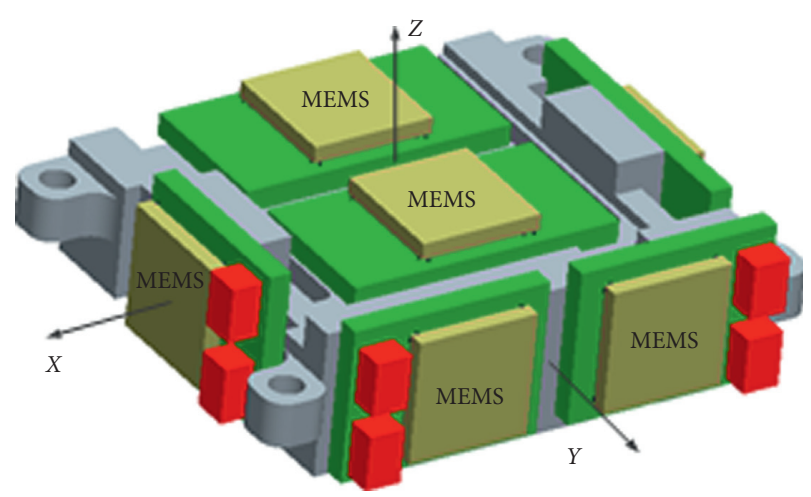

FIGURE 6: MEMS redundant IMU assembly structure based on adhesive joint assembly.

\section{Experiments}

In order to reduce the influence of temperature on installation error and improve the measurement precision of the MEMS-redundant IMU, a novel method of redundant MEMS-IMU based on adhesive process is designed instead of the traditional mechanical assembly technique. The thermal vacuum experiments are performed to compare the influence of temperature on the installation error under the traditional and the new assembly method to verify the improvement of performance by the new assembly method.

4.1. The Thermal Vacuum Experiment of Traditional Mechanical. The angular velocity of the MEMS gyroscope varies with the residual thermal stress produced by the thermal vacuum experiment or the strain resulting from temperature changes. The paper analyzes the influence of thermal vacuum experiment on the bias of MEMS gyroscope under traditional mechanical installation.

The thermal vacuum experiment condition is that the vacuum degree is not less than $6.65 \times 10^{-3} \mathrm{~Pa}$, and the temperature range is $-25^{\circ} \mathrm{C}$ to $50^{\circ} \mathrm{C}$. In the experiment, the temperature was raised from room temperature to $50^{\circ} \mathrm{C}$ and kept for 6 hours. Then, the temperature was reduced to $-25^{\circ} \mathrm{C}$ at a temperature rate of not less than $1^{\circ} \mathrm{C} / \mathrm{min}$ and kept at $-25^{\circ} \mathrm{C}$ for 6 hours. Finally, it returned to room temperature as an experimental cycle. The $X$ axis of MEMS redundant IMU was placed on the platform, and the static drift of the gyroscope was measured for 1 hour before and after the experiment. Before the experiment, the measurement results were measured on the marble platform at normal temperature and pressure. After the experiment, the second measurement results were obtained on the marble platform under the same conditions after the MEMS redundant IMU was placed for one night. It is same way for the $Y$ axis and $Z$ axis. The bias of the gyroscope is calculated by the following formula:

$$
b=\left(\frac{1000 P}{K T} * 3600-7.7376\right)
$$




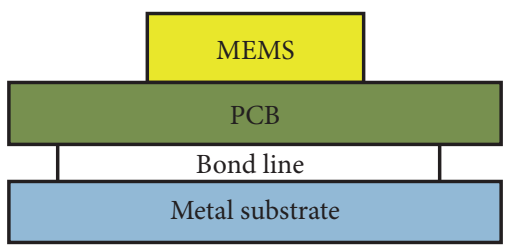

FIgURE 7: Single-axle adhesive joint assembly structure.

where $b$ is the bias of the gyroscope in $\operatorname{deg} / \mathrm{hr}, P$ is the output of angular increment of the MEMS redundant IMU, $T$ is the integration time and it is $\mathrm{ms}$, and $K$ is the scale factor.

The bias measured before and after the experiment is shown in the following table.

As shown in Table 4, the bias of six gyroscopes in the MEMS redundant IMU changes greatly before and after thermal vacuum experiment, and the maximum change is $6.9^{\circ} / \mathrm{h}$ after $100 \mathrm{~s}$ smoothing. The mechanically installed MEMS gyroscope has a large deformation and residual thermal stress in the thermal vacuum experiment. Therefore, the thermal vacuum experiment has a great influence on the bias of MEMS gyroscope installed mechanically.

In the thermal vacuum experiment, the MEMS redundant IMU is placed statically, and each axial gyroscope can only be sensitive to the rotation of the Earth. The measurement error of the MEMS redundant IMU in the mechanical assembly method can be calculated from equation (13) and Table 3. The results are shown in Table 5.

It can be seen from Table 5 when mechanical assembly method is adopted, the maximum measurement error of MEMS redundant IMU can reach $6.9083^{\circ} / \mathrm{h}$.

4.2. The Thermal Vacuum Experiment of Adhesive Joint Assembly. The influence of thermal vacuum experiment on the bias of MEMS gyroscope with adhesive mounting mode is analyzed below. The bias measured before and after the experiment is shown in the Table 6.

The bias of the six MEMS gyroscopes in the MEMS redundant IMU have slight changes before and after the thermal vacuum experiment, and the changes are all less than $0.21^{\circ} / \mathrm{h}$ from Table 6 . Therefore, the thermal vacuum experiment has little effect on the bias of MEMS gyroscope installed with adhesive.

In the calculation of mechanical assembly method, the measurement error of MEMS redundant IMU is shown in Table 7.

As shown in Table 7, when the assembly bonding method is used the measurement error of MEMS redundant IMU does not exceed $0.2079^{\circ} / \mathrm{h}$.

The measurement errors of MEMS redundant IMU between traditional assembly and adhesive joint assembly shown in Table 5 and Table 7 were compared, and calculated the error each other. The specific results are shown in Figure 8 and Table 8.
TABLE 4: Bias comparison of gyroscope combinations $(\% / h)$.

\begin{tabular}{lccc}
\hline Gyro no. & Preexperiment & Postexperiment & Bias \\
\hline$X 1$ & 10.9 & 8.9 & 2 \\
$Y 2$ & -18.2 & -17.4 & -0.8 \\
$Z 3$ & -1.0 & -7.9 & 6.9 \\
$X 4$ & -1.2 & 1.4 & -2.6 \\
$Y 5$ & -4.3 & -4.0 & -0.3 \\
Z6 & -13.5 & -7.3 & -6.2 \\
\hline
\end{tabular}

TABLE 5: Measurement error of mechanical assembly $(\% / \mathrm{h})$.

\begin{tabular}{lc}
\hline Gyro no. & Measurement error \\
\hline$X 1$ & 2.0166 \\
$Y 2$ & -0.8184 \\
$Z 3$ & 6.9083 \\
$X 4$ & -2.6950 \\
$Y 5$ & -0.3574 \\
$Z 6$ & -6.2711 \\
\hline
\end{tabular}

TABLE 6: Comparison of bias of gyroscope combination before and after experiment $\left({ }^{\circ} / \mathrm{h}\right)$.

\begin{tabular}{lccc}
\hline Gyro no. & Preexperiment & Postexperiment & Bias \\
\hline X1 & 6.5175 & 6.6565 & 0.139 \\
Y2 & 2.5871 & 2.4241 & -0.163 \\
Z3 & 12.8579 & 12.7967 & -0.0612 \\
X4 & 10.5128 & 10.6237 & -0.1109 \\
Y5 & 10.0643 & 9.9409 & -0.1234 \\
Z6 & 9.7359 & 9.9413 & 0.2054 \\
\hline
\end{tabular}

TABle 7: Measurement error of adhesive assembly $(\% / h)$.

\begin{tabular}{lc}
\hline Gyro no. & Measurement error \\
\hline$X 1$ & 0.154 \\
$Y 2$ & -0.181 \\
$Z 3$ & -0.1442 \\
$X 4$ & -0.2059 \\
$Y 5$ & -0.1808 \\
$Z 6$ & 0.2079 \\
\hline
\end{tabular}

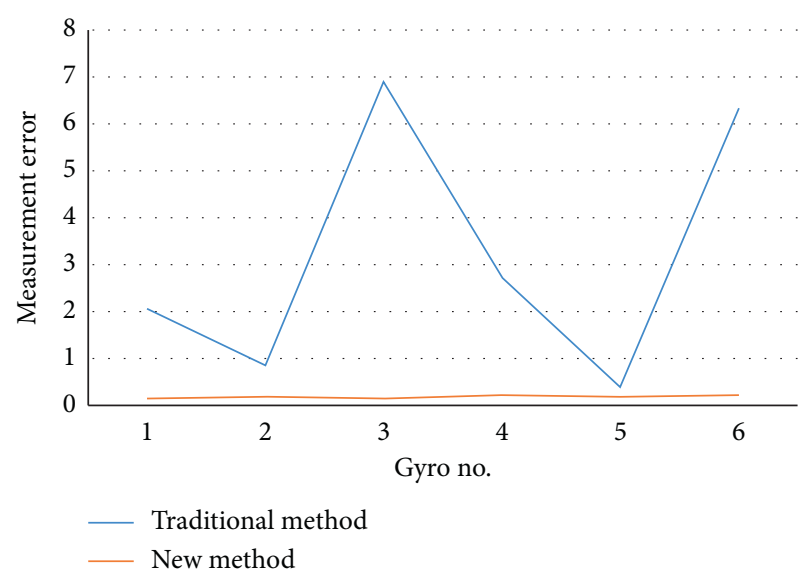

FIgURE 8: Comparison of measurement precision between traditional method and adhesive joint assembly $(\% / h)$. 
TABle 8: Comparison of measurement precision between traditional method and adhesive joint assembly $(\% / h)$.

\begin{tabular}{lcc}
\hline Gyro no. & Measurement error difference & $\begin{array}{c}\text { Measurement error } \\
\text { ratio }\end{array}$ \\
\hline$X 1$ & 1.8626 & 13.09481 \\
$Y 2$ & -0.6374 & 4.521547 \\
$Z 3$ & 7.0525 & -47.9078 \\
$X 4$ & -2.4891 & 13.08888 \\
$Y 5$ & -0.1766 & 1.97677 \\
$Z 6$ & -6.479 & -30.164 \\
\hline
\end{tabular}

From Table 8, the measurement error between traditional method and adhesive joint assembly can be up to $7.0525^{\circ} / \mathrm{h}$, and the measurement precision can be increased by 47 times at most. Therefore, compared with the traditional assembly method, the adhesive joint assembly can improve the measurement precision of MEMS redundant IMU by an order of magnitude.

\section{Conclusion}

In this paper, an optimization method of MEMS redundant IMU based on adhesive joint assembly is proposed to improve the precision of MEMS redundant IMU. The paper analyzed the actual application environment of the MEMS gyroscope and selected the adhesive materials matching the material thermo physical parameters with the circuit board. By optimizing the bonding process and designing a reasonable bonding layer structure, the installation errors of each axes of MEMS redundant IMU under different temperature are better than those of the traditional mechanical connection. The experimental results confirm that the bias affected by temperature is not more than $0.21^{\circ} / \mathrm{h}$ with the new assembly method. Compared with the traditional method, the precision of MEMS redundant IMU in the new assembly method is improved by an order of magnitude.

\section{Data Availability}

The raw/processed data required to reproduce these findings cannot be shared at this time as the data also forms part of an ongoing study.

\section{Conflicts of Interest}

We declare that we do not have any commercial or associative interest that represents a conflict of interest in connection with the work submitted.

\section{Acknowledgments}

This work was partially sponsored by Program of Shanghai Academic/Technology Research Leader under Project 18XD1421700, the Natural Science Foundation of Shaanxi under Project 2020JM-488, and Shanghai Rising Star Program under 20QA1404300.

\section{References}

[1] Y. A. Nekrasov, N. V. Moiseev, Y. V. Belyaev, S. V. Pavlova, and R. G. Lyukshonkov, "Influence of translational vibrations, shocks and acoustic noise on MEMS gyro performance," Gyroscopy and Navigation, vol. 8, no. 1, pp. 31-37, 2017.

[2] Z. Xueting and X. U. Huan, "Research on MEMS gyro error correction algorithm based on fuzzy kalman," Journal of Hangzhou Dianzi University (Natural ences), vol. 39, no. 1, pp. 1-6, 2019.

[3] L. I. Wei-zhong and P. SUN, "Research on progress and the key technology of MEMS gyroscope in tactical missile," Navigation and Control, vol. 16, no. 3, pp. 97-104, 2017.

[4] C. Zhang, S. Li, S. Gao et al., "Design of MEMS strapdown inertial system based on FPGA," Piezoelectrics \& Acoustooptics, vol. 39, no. 2, pp. 176-179, 2017.

[5] G. Yan, Strapdown Inertial Navigation Algorithm and Integrated Navigation Principles, Northwestern Polytechnical University Press, Xi'an, China, 2019.

[6] G. Hu, L. Ni, B. Gao et al., "Model predictive based unscented kalman filter for hypersonic vehicle navigation with INS/ GNSS integration,” IEEE Access, vol. 8, pp. 4814-4823, 2020.

[7] J. Cheng, J. Dong, R. Landry, and D. Chen, "A novel Optimal configuration form redundant MEMS inertial sensors based on the orthogonal rotation method," Sensors, vol. 14, no. 8, pp. 13661-13678, 2014.

[8] C. Jianhua, M. Hongjie, S. Xiangyu et al., "Dual-axis rotational modulation method based on SINS tetrahedron redundancy configuration," Systems Engineering and Electronics, vol. 39, no. 8, pp. 1800-1807, 2017.

[9] K. He, J. Han, and Y. Shao, "A novel redundant inertial measurement unit and calibration algorithm," in Proceedings of the Optoelectronics and Microelectronics (ICOM), 2013 International Conference, Harbin, China, September 2013.

[10] W. Zhou, X. Li, and S. Zhao, "Fault-tolerant algorithm and information management for redundant strapdown inertial navigation system," Journal of National University of Defense Technology, vol. 40, no. 1, pp. 32-36, 2018.

[11] A. Nazemipour, M. T. Manzuri, D. Kamran et al., "MEMS gyro bias estimation in accelerated motions using sensor fusion of camera and angular-rate gyroscope," IEEE Transactions on Vehicular Technology, vol. 69, no. 4, pp. 3841-3851, 2020.

[12] L. I. Xun, X. Zhang, S.-d Sun et al., “Alignment experiments and analysis of error model for MEMS gyroscope," Electronic Design Engineering, vol. 25, no. 17, pp. 164-167, 2017.

[13] H. Yang, H. Jiang, M. A. Xin et al., "Application of improved wavelet thresholding method and extreme learning machine in MEMS gyro random errors compensation," Chinese Journal of Sensors and Actuators, vol. 31, no. 10, pp. 79-82, 2018.

[14] W. U. Bao-feng, X. I. A. Lin-yuan, D.-j. Wu, and H.-y. Zhu, "Random error analysis and wavelet threshold denoising of MEMS gyroscope," Electronic Design Engineering, vol. 28, no. 15 , pp. $1-5,2020$.

[15] P. Zhang, X. Zhan, X. Zhang et al., "Error characteristics analysis and calibration testing for MEMS IMU gyroscope," Aerospace Systems, vol. 2, pp. 97-104, 2019.

[16] U. Qureshi and F. Golnaraghi, "An algorithm for the in-field calibration of a MEMS IMU,” IEEE Sensors Journal, vol. 17, no. 22, pp. 7479-7486, 2017.

[17] C. Jianhua, L. Ping, G. Peng, M. Zou, and W. Fu, "Highprecision calibration scheme for RIMU," IEEE Access, vol. 7, pp. 72376-72386, 2019.

[18] J. Sun, J. Zou, and T. Hu, "A fast calibration method for MEMS inertial sensors based on 24-position," Piezoelectrics \& Acoustooptics, vol. 41, no. 3, pp. 440-444, 2019. 
[19] Y. A. N. Xue-ping, Li Cheng, Q.-f Han et al., "Package materials and processes of MEMS devices," Semiconductor Technology, vol. 31, no. 12, pp. 900-903, 2006.

[20] Z. Gan, Research on Vacuum Packaging of MEMS devices, Huazhong University of Science \& Technology, Wuhan, China, 2008. 